\title{
Rhabdomyosarcoma - site matters
}

\author{
P J Greyling, MB ChB, BSC (Hons) \\ S Andronikou, MB BCh, FCRad, FRCR, PhD \\ C Ackermann, MB ChB, MMed Rad (D), MRCS \\ O C Schulze, $M B C h B$
}

Stellenbosch University, Tygerberg Hospital

\section{N A Wieselthaler, MB ChB, FCRad}

University of Cape Town, Red Cross Hospital

\begin{abstract}
For the clinical radiologist imaging a soft-tissue mass in a child, site plays an important role in narrowing the differential diagnosis. Rhabdomyosarcomas can arise from any tissue, even those devoid of skeletal muscle. Knowledge of the common sites involved allows inclusion in the differential diagnosis where relevant and also has a limited potential to prognosticate. With this article we hope to give some clarity as to when this diagnosis is likely. Rhabdomyosarcomas are relatively rare, but radiologists should have a high index of suspicion at particular sites where this may be the most common childhood malignancy. Common sites are the head and neck, genito-urinary system and extremities.
\end{abstract}

\section{Introduction}

Radiologists are often unsure whether a rhabdomyosarcoma should be included in a differential diagnosis of a solid mass found on imaging.
With this article we hope to give some clarity as to when this diagnosis is likely. Rhabdomyosarcomas are relatively rare, but radiologists should have a high index of suspicion at particular sites where this may be the most common childhood malignancy. Rhabdomyosarcoma is the most common soft-tissue sarcoma (50\% of soft-tissue sarcomas) $)^{1}$ of childhood, representing $5 \%$ of all childhood cancers. ${ }^{2}$

Rhabdomyosarcoma is the third most common extracranial solid tumour in children, after neuroblastoma and Wilm's tumour. ${ }^{3}$ These tumours are thought to arise from primitive mesenchymal cells committed to skeletal muscle differentiation and can occur in a variety of organs and tissues, including those that lack striated muscle, ${ }^{2}$ as well as from smooth-muscle cells, i.e. urinary bladder. ${ }^{4}$ Tables I and II summarise the incidence of these tumours at specific sites and the prognosis related to the site involved.

\begin{tabular}{|lc|}
\hline & Table I. Sites of primary tumours \\
\hline Site & \% of patients \\
\hline Head and neck & 35 \\
• Orbit & 10 \\
• Parameningeal & 10 \\
• Other & 15 \\
Genito-urinary & 26 \\
Extremities & 19 \\
Other & 20 \\
\hline
\end{tabular}

\begin{tabular}{|ll|}
\hline \multicolumn{1}{|c|}{ Table II. Favourable and unfavourable sites } \\
\hline Favourable & \multicolumn{1}{c|}{ Unfavourable } \\
\hline Non-parameningeal head and neck sites & Parameningeal head and neck sites \\
$\bullet$ Oropharynx & $\bullet$ Middle ear \\
$\bullet$ Scalp & • Nasal cavity \\
$\bullet$ Parotid & $\bullet$ Paranasal sinuses \\
$\bullet$ Neck & • Nasopharynx \\
$\bullet$ Larynx & $\bullet$ Infratemporal fossa \\
$\bullet$ Cheek & • Pterygo-pallatine fossa \\
$\bullet$ Hypopharynx & • Parapharyngeal region \\
Genito-urinary system (other than bladder and prostate) & Orbits involving base of skull \\
Orbits and eyelids & Trunk \\
& Bladder and prostate \\
& 'Other' sites \\
\hline
\end{tabular}




\section{PICTORIAL REVIEW}

\section{Head and neck}

Primary head and neck tumours are most commonly diagnosed in children younger than 8 years of age. ${ }^{4}$

\section{Non-parameningeal}

Orbital. Overall orbital tumours have the best prognosis. ${ }^{1}$ Orbital tumours are generally non-invasive and confined to the bony orbit. ${ }^{5}$ Orbital tumours with intracranial invasion or bone destruction are for practical purposes treated as parameningeal disease ${ }^{5}$ (Figs 1a and b).

\section{Parameningeal regions and paranasal sinuses}

Rhabdomyosarcoma is the most common malignancy to arise within the nasal cavity or paranasal sinuses in children. ${ }^{3}$ Tumours tend to be large and invasive at these sites. ${ }^{5}$ Sites include the nasopharynx, paranasal sinuses, middle ear, mastoid and pterygoid and infratemporal sites. Tumours in parameningeal sites usually behave more aggressively than do tumours in other parts of the head and neck, primarily because of the proximity to the meninges and brain. ${ }^{6}$ Rhabdomyosarcomas of the head and neck grow insidiously and often invade intracranial space through the numerous foramina leading to the brain (Figs $2 \mathrm{a}-\mathrm{d}$ ).

Temporal bones (Fig. 3). Paediatric rhabdomyosarcomas that arise in the temporal bone (middle ear or mastoid), although rare, are generally considered to be aggressive neoplasms by virtue of their proximity to vital structures, their tendency to spread intracranially, and their potential for meningeal involvement. ${ }^{6}$ Patients with rhabdomyosarcomas of the middle ear and mastoid have a particularly poor prognosis. ${ }^{6}$

\section{Genito-urinary}

Bladder and prostate are the most common genito-urinary sites. ${ }^{4}$ Other common sites involved are the vagina, uterus and paratesticular region. ${ }^{\text {? }}$

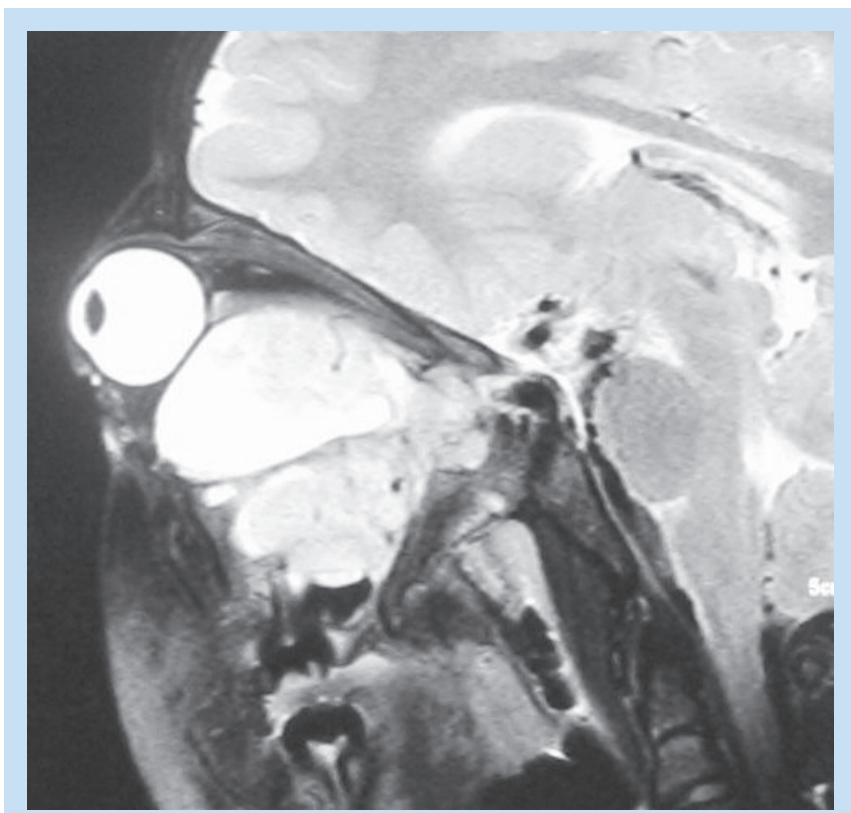

Fig. 1a. Sagittal oblique STIR MRI demonstrates a mass which has displaced globe and optic nerve superiorly. It appears to have expanded the orbit (indicating slow growth) and has also destroyed the orbital floor, extending into the maxillary sinus and unerupted teeth in the alveolar bone.

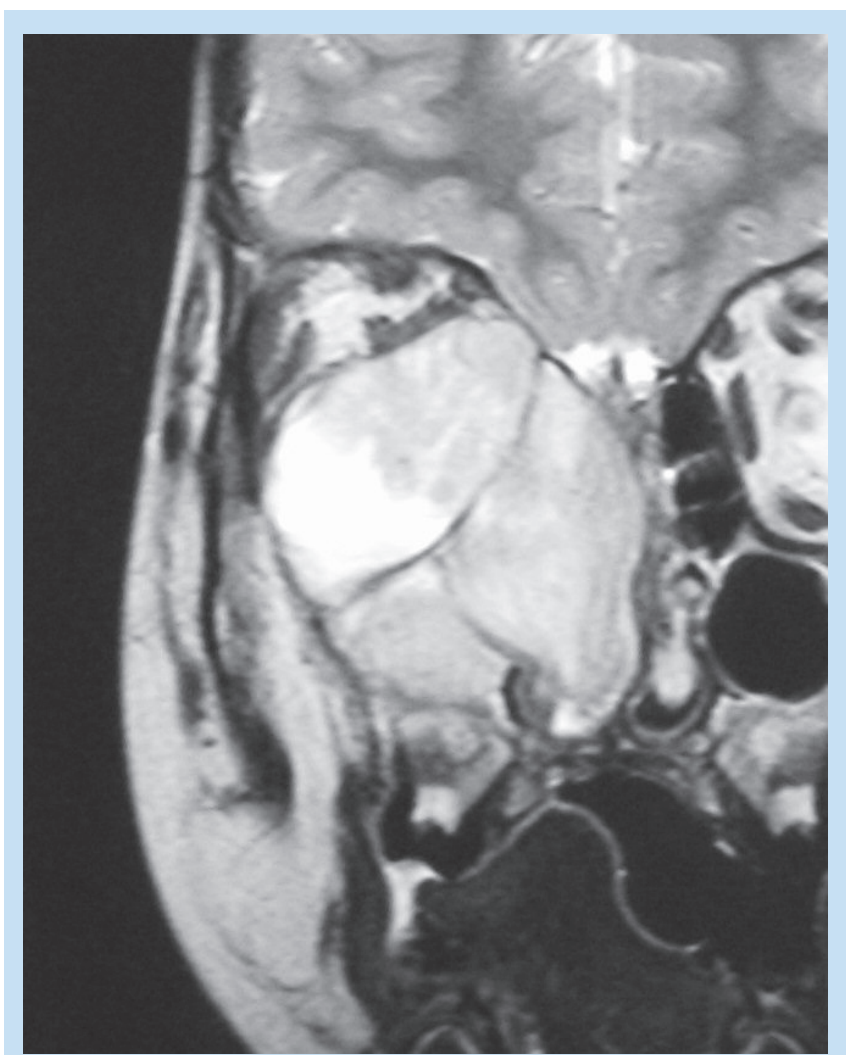

Fig. 1b. Coronal T2-weighted MRI demonstrating a mass near the orbital apex, extending through the lamina paperaceae and orbital floor into the nasal cavity, ethmoid and maxillary sinuses.

Large pelvic masses are common at time of diagnosis. ${ }^{4}$

Most rhabdomyosarcomas of the bladder arise near the trigone or urethral orifice, are of embryonal histology and carry a good prognosis. ${ }^{8}$ Tumours of the bladder and prostate generally have a worse prognosis than other sites such as the vagina. ${ }^{5}$ Tumours originating in the prostate carry a significantly worse prognosis than tumours that involve the bladder only. When both are involved, the site of origin is often difficult to determine $^{2}$ (Fig. 4a and b).

The term paratesticular rhabdomyosarcoma has been applied to primary tumours arising in the spermatic cord, testis, epididymis and penis. ${ }^{5}$ Rhabdomyosarcoma is the most common paratesticular malignancy in children. ${ }^{2}$ Most paratesticular rhabdomyosarcomas are composed of the spindle cell variant of embryonal type and this carries an especially good prognosis. ${ }^{2}$ Prognosis of paratesticular rhabdomyosarcoma is generally good, but worse in children older than 10 years of age or if there is retroperitoneal lymph node involvement. ${ }^{5}$ Paratesticular rhabdomyosarcomas usually occur in adolescent males. ${ }^{1}$

Rhabdomyosarcoma is the most common malignancy of the lower genital tract in young women but has an excellent prognosis. Cervical rhabdomyosarcoma is usually seen in the second decade of life, unlike vaginal lesions which usually present before the age of 4 years. Uterine rhabdomyosarcoma carries a worse prognosis than vaginal lesions. ${ }^{8}$

Prognosis is unfavourable for patients who have locally advanced pelvic tumours for which the exact site of origin cannot be determined. ${ }^{2}$ 


\section{PICTORIAL REVIEW}
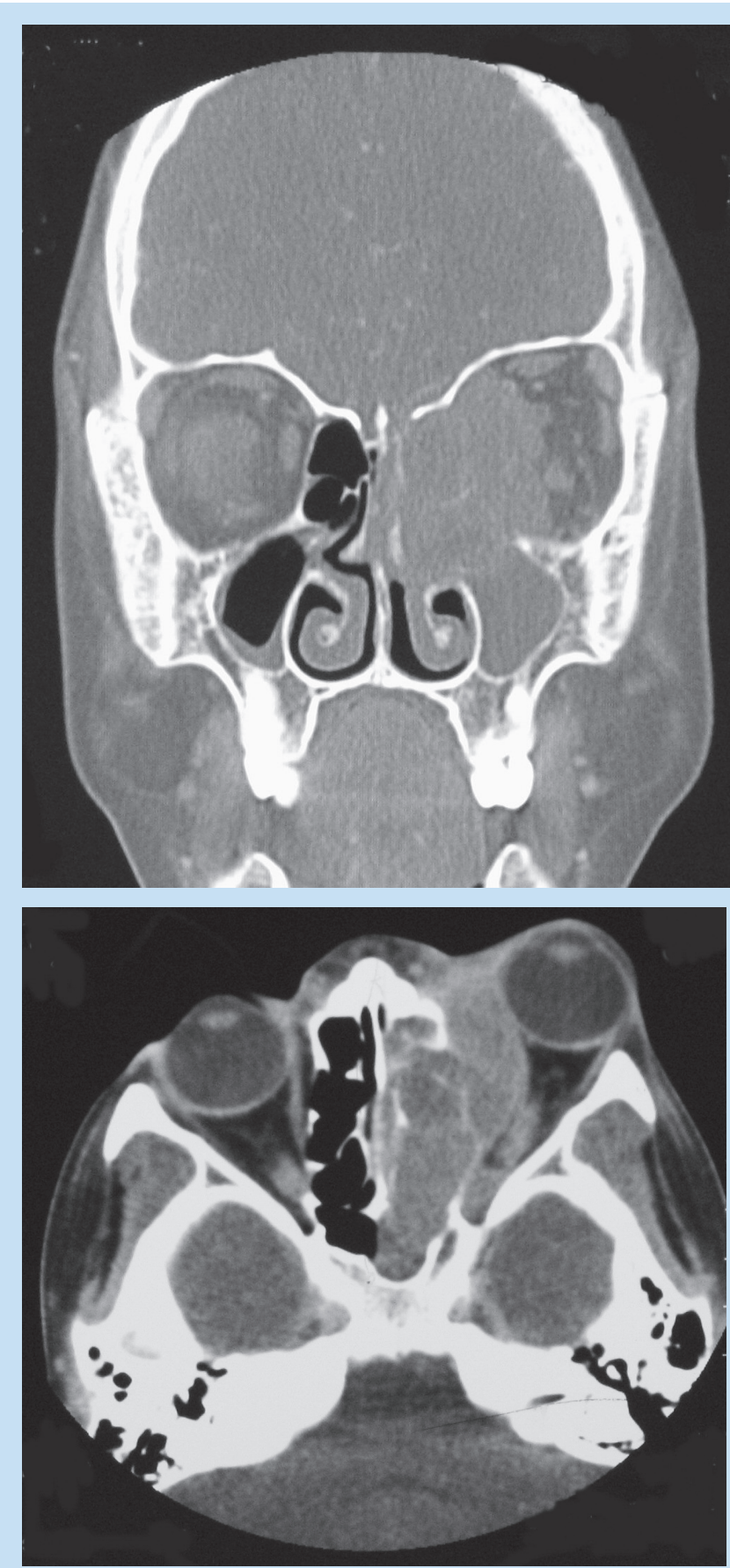

Figs 2a and $b$. Coronal and axial CT scans of paranasal sinuses demonstrating a soft-tissue mass which has destroyed the wall of the ethmoid sinuses, the middle and superior turbinates, and the medial wall and inferior aspect of the orbit. The mass is seen to fill the superior portion of the nasal cavity, the left maxillary sinus and part of the left orbit. The inferior and medial recti are displaced laterally.

\section{Extremities}

Extremity tumours are seen most commonly in adolescents. Approximately half of extremity tumours are of alveolar subtype, accounting in part for the poor outcome in these patients. Lymph node involvement is common and imaging should include the entire nodal basin $^{2}$ (Figs 5a-c).

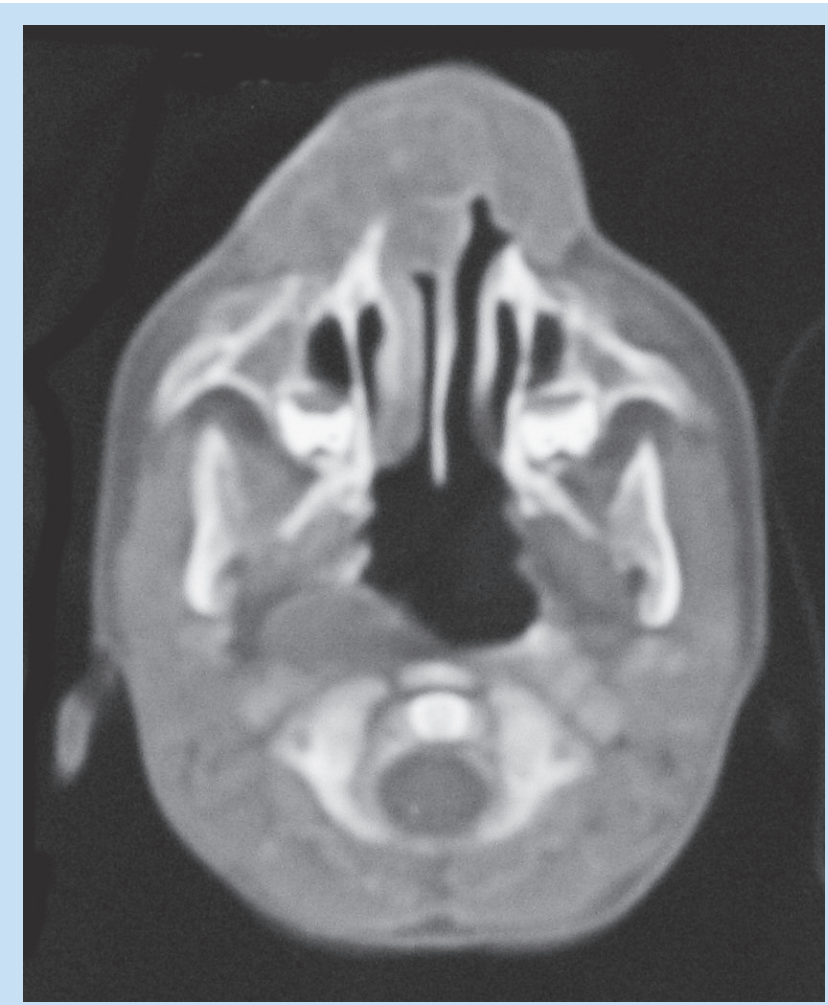

Fig. 2c. CT scan demonstrating a soft-tissue mass involving the anterio aspect of the nose and maxillary bones bilaterally with some extension into the right anterior choana.

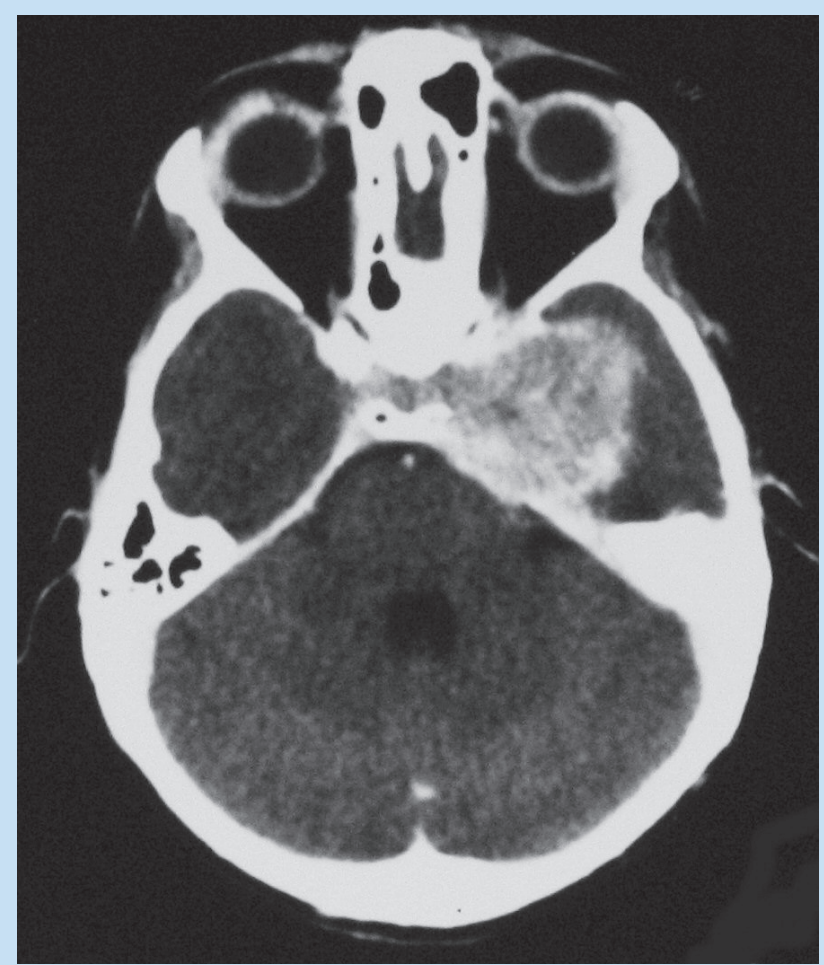

Fig 2d. An 8-year-old boy with rhabdomyosarcoma of the maxillary sinus, middle cranial fossa and base of the skull. Contrast-enhanced CT of the brain demonstrates a non-homogeneous enhancing mass in the left middle cranial fossa, involving the cavernous sinus and pituitary fossa. 


\section{PICTORIAL REVIEW}

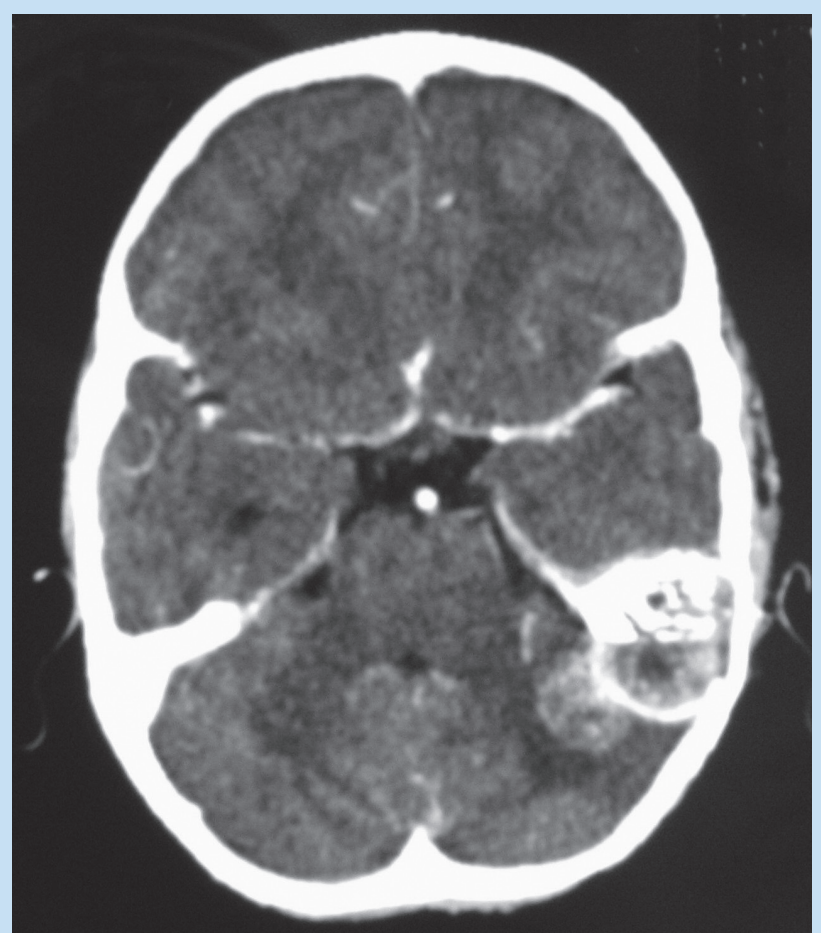

Fig. 3. Axial contrast-enhanced CT scan demonstrating a mass with destruction of the left petrous bone, extending to involve posterior and middle cranial fossa in the form of a soft-tissue mass. The mass also extends into the external ear canal (not shown here).

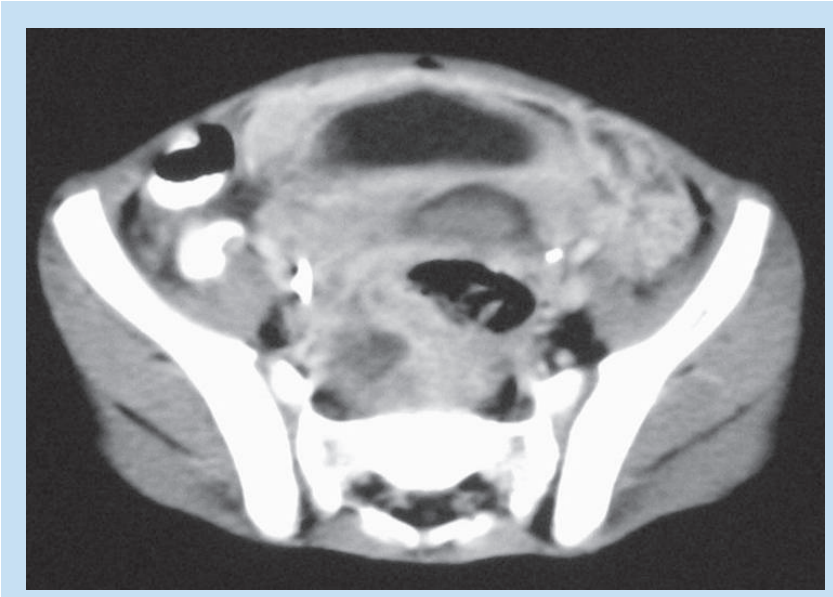

Fig. 4a. A 2-year-old boy with rhabdomyosarcoma in the bladder. T1weighted axial MRI shows the mass to be iso-intense to muscle on T1 (mixed signal on T2). The rectum is seen separately from the mass.

\section{Other sites}

Intrathoracic, retroperitoneal, perineal and peri-anal regions can harbour a primary rhabdomyosarcoma that can remain undiagnosed for a long period of time. These tumours can become very large and metastasise before they are diagnosed. Uncommonly rhabdomyosarcomas arise from the liver, brain, heart, breast or ovary ${ }^{4}$ (Figs 6a and b).

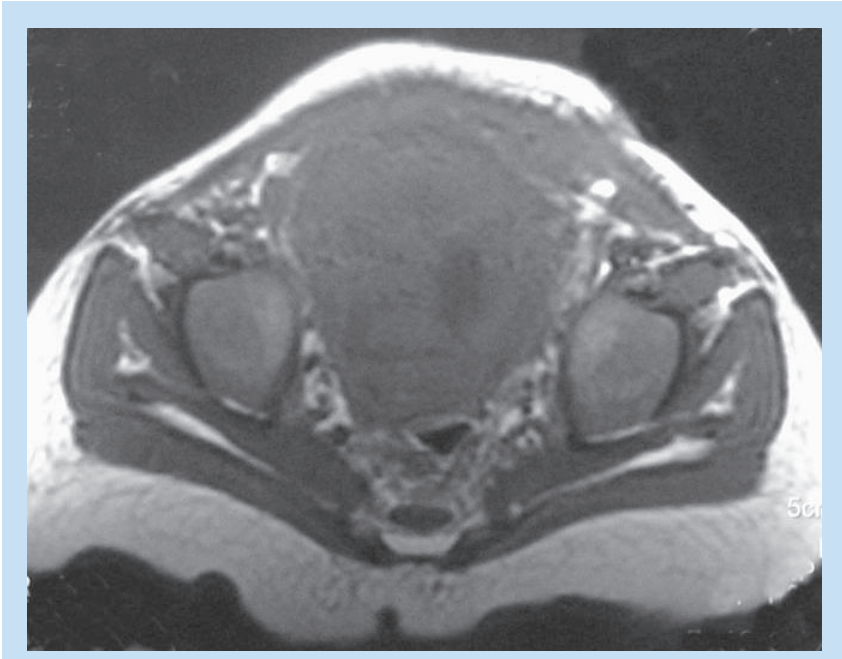

Fig 4b. A 5-year-old girl with rhabdomyosarcoma in the pelvis. Contrastenhanced CT scan of pelvis shows a non-homogeneous enhancing mass presacrally. The mass both encases and displaces the rectum. The tissue planes are also obscured between bladder and rectum.

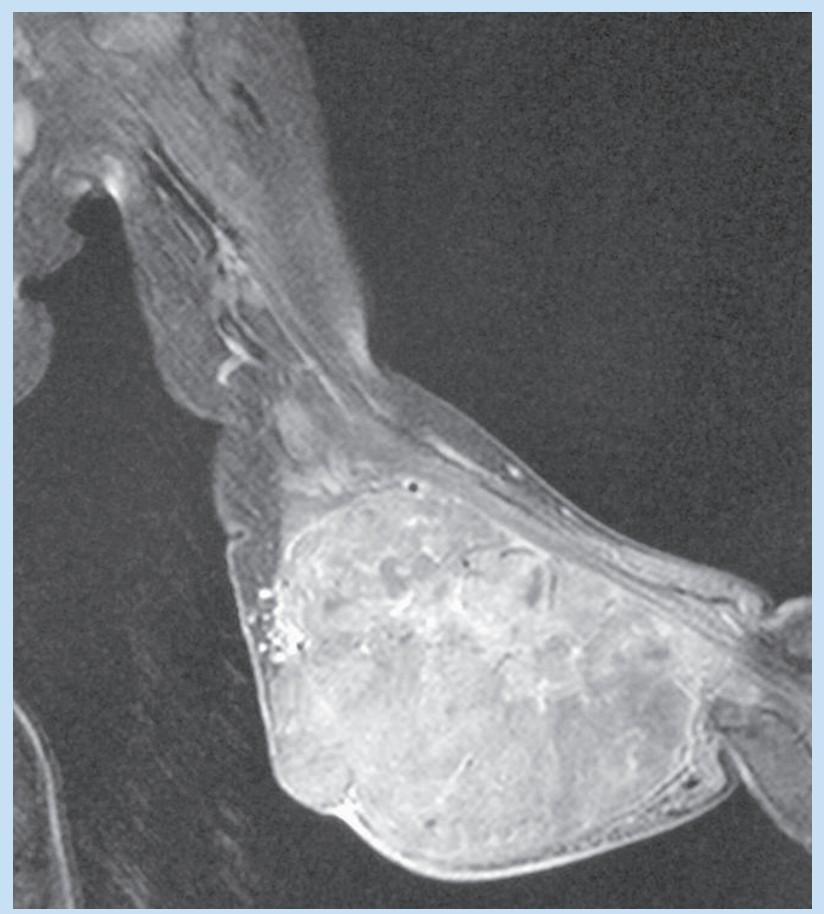

Fig. 5a. A 4-month- old boy with a rhabdomyosarcoma of the right forearm. Short Tau Inversion Recovery MRI of upper limb demonstrates an inhomogeneous, fairly well encapsulated mass of relatively high signal intensity in the forearm. The mass extends to the level of the olecranon but does not cross the joint space. The flexor tendons and vessels are seen separately.

\section{Site-related histology}

Embryonal. This type often arises in mucosal-lined structures of the nasopharynx, auditory canal and genito-urinary and gastro-intestinal tracts. ${ }^{2}$ Embryonal tumours usually occur before age $8 .{ }^{1}$ 


\section{PICTORIAL REVIEW}
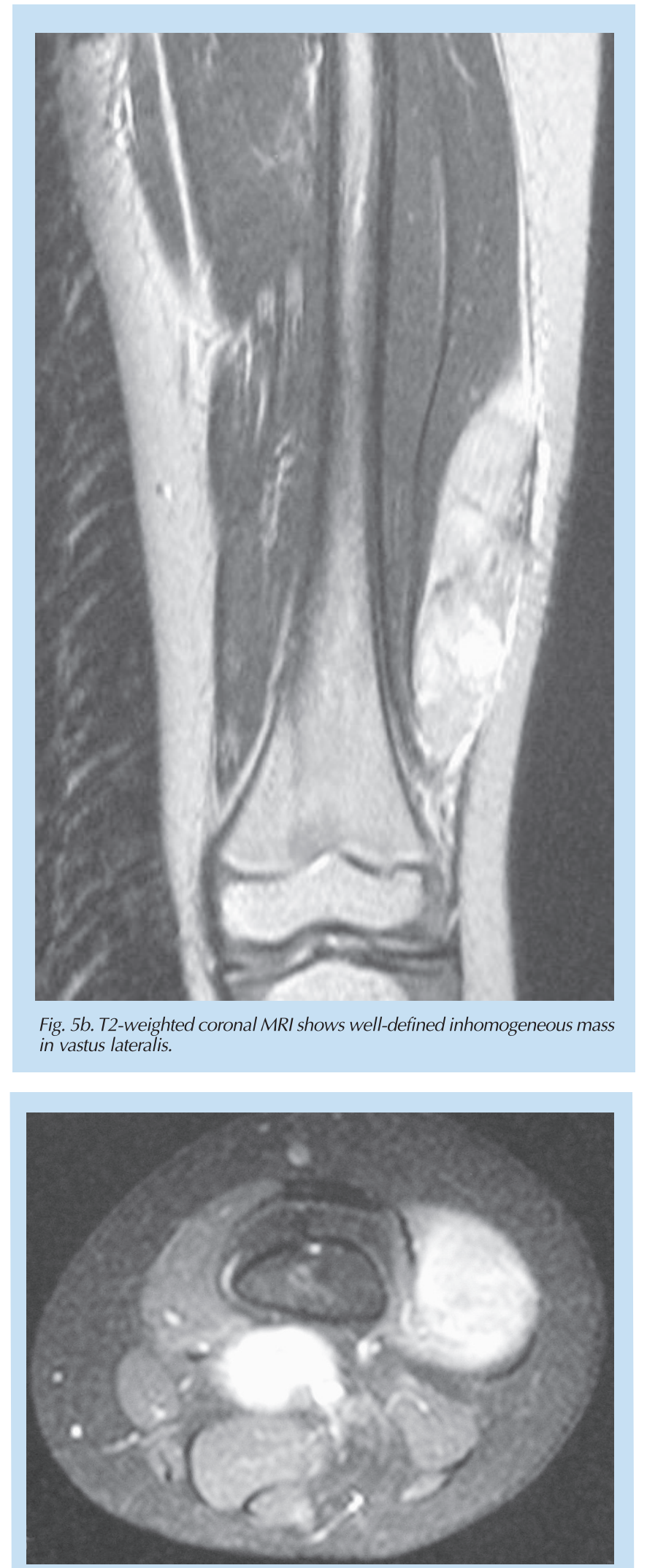

Fig. 5c. T1-weighted fat-saturated gadolinium-enhanced axial MRI shows two masses in distal thigh. Both demonstrate strong enhancement with one mass in the distal vastus lateralis and the second in the popliteal fossa, just anterior to the popliteal artery

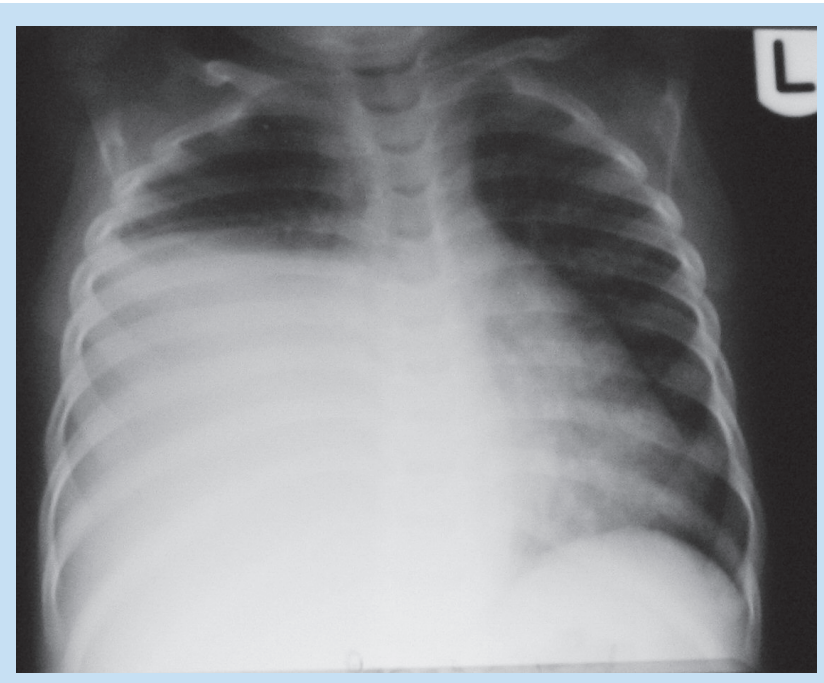

Fig. 6a. A 2-year-old boy with rhabdomyosarcoma of the right chest/abdomen (possibly arising from right diaphragm). Chest X-ray shows right-sided chest mass resulting in 'apparent' elevation of right hemidiaphragm, with displacement of heart to left.

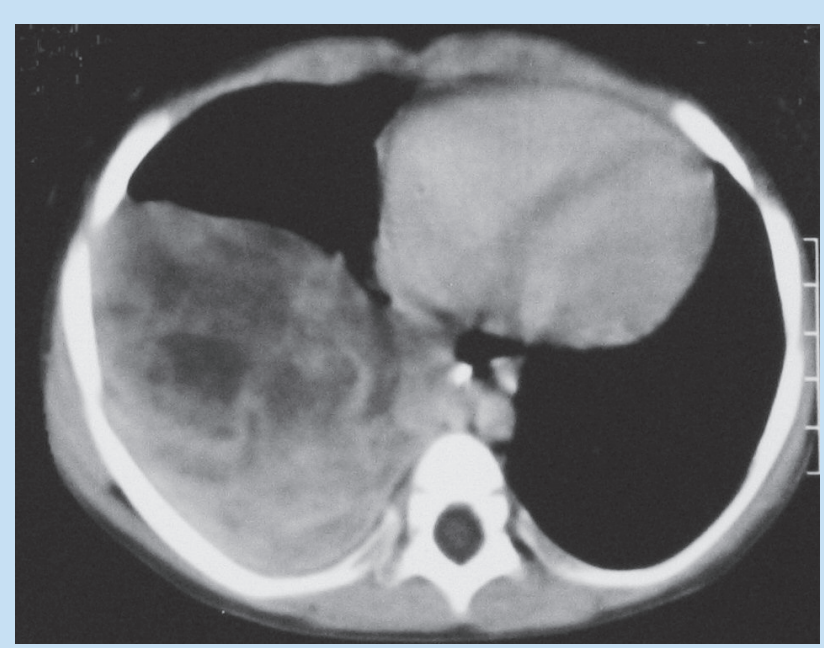

Fig. 6b. Contrast-enhanced CT of chest/abdomen shows large heterogeneous mass in right hemithorax. There are low density areas in mass compatible with tumor breakdown, and pleural thickening is also visible.

Botryoid. A variant of embryonal type occurs in hollow cavities such as the vagina, biliary tract and nasopharynx. ${ }^{1}$

Alveolar. Most commonly these occur on the trunk and extremities (and have a worse prognosis). ${ }^{1}$

\section{Metastases}

Rhabdomyosarcoma commonly metastasises to lungs (36\%), bone marrow $(22 \%)$ or cortical bone $(7 \%)^{2}$ (Fig. 7$)$.

\section{Conclusion}

For the clinical radiologist imaging of a soft-tissue mass in a child plays an important role in narrowing the differential diagnosis. Rhabdomyosarcomas can arise from any tissue, even those devoid of skeletal muscle. Knowledge of the common sites involved allows inclu- 


\section{PICTORIAL REVIEW}

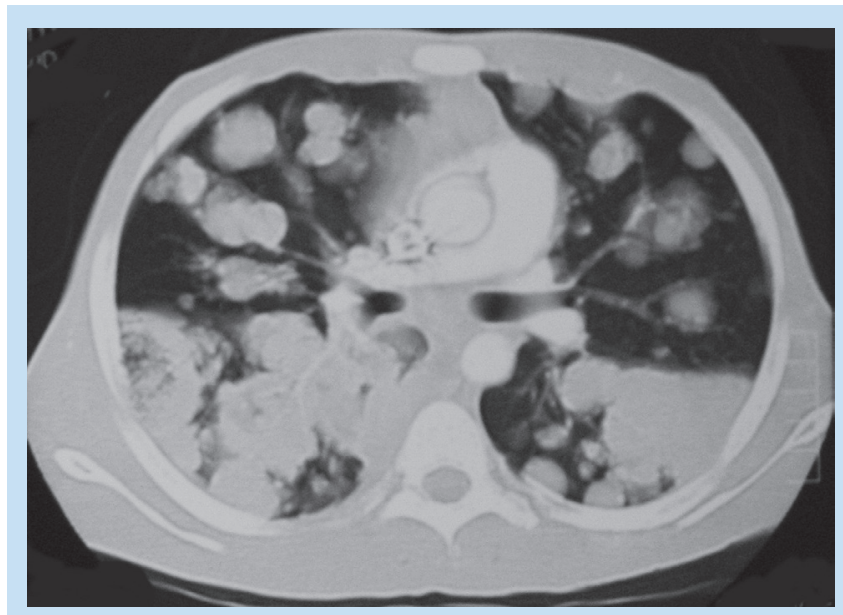

Fig. 7. Lung metastases on CT due to a pelvic rhabdomyosarcoma. sion in the differential diagnosis when relevant, and also has a limited potential in determining prognosis.

1. Stuart A, Radhakrishnan J. Rhabdomyosarcoma. Indian J Pediatr 2004; 71: 331-337.

2. McCarville M, Spunt S, Pappo A. Rhabdomyosarcoma in pediatric patients. the good, the bad, and the unusual. AJR 2001; 176: 1563-1569.

3. Hermann B, Sotela-Avila C, Eisenbeis J. Pediatric sinonasal rhabdomyosarcoma: three cases and a review of literature. Am J Otolaryngol 2003; 24: 174-180.

4. Kline N, Sevier N, Solid tumors in children. J Pediatr Nurs 2003; 18(2): 96-102.

5. McHugh K, Boothroyd A. The role of radiology in childhood rhabdomyosarcoma. Clin Radiol 1999; 54 $2-10$

6. Abbas A, Awan S. Rhabdomyosarcoma of the middle ear and mastoid: A case report and review of the literature. Ear Nose Throat J 2005; 84: 780.

7. Humpl T, Thuroff J, Gutjahr P. Paediatric urological malignancies. BJU Int 2001; 87: 741-746.

8. Behtash N, Mousavi A, Tehranian A, Khanafshar N, Hanjani P. Embryonal rhabdomyosarcoma of the uterine cervix: case report and review of the literature. Gynecol Oncol 2003; 91: 452-455.

\section{Internal Derangements of Joints, 2-Volume Set, 2nd edition}

\begin{tabular}{|l|l|l|}
\hline $\begin{array}{l}\text { Internal } \\
\text { Derangements } \\
\text { of Joints }\end{array}$ & $\begin{array}{l}\text { To Order Contact: } \\
\text { Health \& Medical Publishing } \\
\text { Group } \\
\text { Private Bag X1, Pinelands, 7430 } \\
\text { Tel: 021 - 6578200 Fax: 021- } \\
6834509 \\
\text { e-mail: carmena@hmpg.co.za / } \\
\text { brents@hmpg.co.za }\end{array}$ \\
\hline
\end{tabular}

\section{ISBN $0721695523 \cdot$ Hardback · 2400 Pages $\cdot 2859$ Illustrations Saunders · Forthcoming Title (December 2006)}

Donald Resnicks latest reference keeps pace with the rapid changes that characterize modern MR imaging of joints This 2nd edition offers comprehensive coverage of the most up-to-date protocols and imaging techniques required in the analysis of internal derangements of the six major peripheral joints: the shoulder, elbow, wrist and hand, pelvis and hip, knee, and ankle and foot. Youll find the level of utility you need through the new, streamlined organization that explores MRI Techniques and Protocols; Synovial Joints: General Concepts; Bone and Bone Marrow; Soft Tissues; and Specific Joints in five new, easy-to-reference sections. Youll also find hundreds of new illustrations " new expertly designed anatomic diagrams " and updated radiographic and CT images correlated with the latest highresolution MR images as appropriate.

\section{New in this edition}

" Dedicates all-new chapters to the hottest topics in the field: Infectious Disorders of Bone and Joint " Ischemic Disorders of Bone " Osteoporosis " Tumors and Tumor-like Disorders of Bone " Disorders of Ligaments.

" Features a new organization that makes important information easy to find.

" Provides hundreds of new images to clarify every discussion and every technique.

" Presents the latest MRI protocols for all major peripheral joints of the body.

" Correlates the latest high-resolution MR images to relevant radiographic and CT images.

" Offers brand-new anatomic diagrams from master medical illustrator, Michael Stadnick and hundreds of new images from the private collection of co-author, Michael Pretterklieber 